\title{
Hipertensão arterial e hipertrofia ventricular esquerda em diabetes mellitus tipo 2
}

\author{
Consuelo Padilha Villar ${ }^{1}$ \\ Luciana G. Matteoni de Athayde ${ }^{2}$ \\ Maria das Dores Acioli de Lima ${ }^{3}$
}

\begin{abstract}
Resumo
Para avaliar a prevalência de hipertensão arterial sistêmica (HAS) e da hipertrofia ventricular esquerda (HVE), bem como a associação entre ambas, em pacientes com diabetes mellitus tipo 2 (DM2), foram estudados 204 pacientes atendidos no Hospital Universitário Alcides Carneiro (HUAC) da Universidade Federal de Campina Grande - PB. Os pacientes apresentavam uma média de idade de 58,0 $\pm 11,4$ anos, sendo $64,2 \%$ ( $n=131 / 204)$ do gênero feminino. A HAS esteve presente em 66,7\% ( $\mathrm{n}=136 / 204)$ da amostra estudada, sendo mais freqüente nas mulheres (69,5\%), nos indivíduos do grupo racial branco e ex-tabagistas. Observou-se uma associação estatisticamente significante entre HAS e a idade $(p<0,0001)$, o tempo de evolução do diabetes $(p<0,015)$, o tabagismo $(p<0,009)$ e o aumento da circunferência abdominal $(p<0,008)$. A HVE esteve presente em 11,3\% (23/204) dos pacientes, quando diagnosticada por critérios eletrocardiográficos. O ecocardiograma (ECO), realizado em 23\% (47/204) dos pacientes, mostrou uma prevalência de HVE de 76,6\% (36/47) e, apesar de ocorrer mais em hipertensos (85,2\%) que nos não-hipertensos (65,0\%), ao se avaliar a relação entre HAS e HVE, observou-se não existir associação estatisticamente significante entre essas variáveis, na amostra estudada $(p>0,19)$. Concluiu-se que a prevalência de HAS foi de $66,7 \%$, na população estudada, relacionandose à idade, ao tempo de evolução do diabetes, ao tabagismo e ao aumento da circunferência abdominal. A HVE mostrou também uma elevada prevalência $(76,6 \%)$ quando avaliada utilizando-se o $\mathrm{ECO}$, porém não relacionada de forma estatisticamente significante à HAS.
\end{abstract}

Palavras-chave: hipertensão arterial; hipertrofia ventricular esquerda; diabetes tipo 2.

\section{INTRODUÇÃO}

O aumento da sobrevida da população tornou mais aparente a importância das doenças crônicas, incluindo a hipertensão arterial sistêmica (HAS) e o diabetes mellitus tipo 2 (DM2), como grandes desafios para a Saúde Pública.

A HAS é uma doença de elevada prevalência, que acomete aproximadamente
$20 \%$ da população em países industrializados (BRANDÃO et al., 2000). No Brasil, segundo o Ministério da Saúde, 34\% dos óbitos foram associados a doenças cardiovasculares. Nesse contexto, a HAS acomete, no País, de 15\% a $20 \%$ da população adulta (III CONSENSO..., 1998). Por sua vez, o DM2 na população geral

\footnotetext{
${ }^{1}$ Pós-graduanda do Mestrado Interinstitucional UFAC/UFBA. Rio Branco - AC

${ }^{2}$ Acadêmica de Medicina da UFBA. Bolsista do Programa de Educação Tutorial (PET). Salvador - BA

${ }^{3}$ Professora Adjunto do Departamento de Medicina da Faculdade de Medicina - UFBA. Salvador - BA

Correspondência para / Correspondence to:

Maria das Dores Acioli de Lima

Luciana G. Matteoni de Athayde

Ambulatório Magalhães Neto. Rua Padre Feijó, $3^{\circ}$ andar - Canela.

40.110-170 Salvador - Bahia - Brasil.

Tel: (0xx71) 3203-2744, ramal 36.

E-mail:lgmatteoni@yahoo.com
} 
tem prevalência de 7,6\% (MALERBI; FRANCO, 1992).

A associação entre HAS e DM2 é encontrada freqüentemente na prática clínica, sendo a prevalência $(40 \%$ a $50 \%)$ consideravelmente maior que na população geral (WEIDMANN; BOEHLEN; DE COURTEN, 1993). Entre diabéticos com idade superior a 75 anos, $60 \%$ são portadores de HAS (CONTI, 2001). As pessoas diabéticas têm, portanto, chance duas a três vezes maior de apresentar HAS.

Por sua vez, o DM2 está associado ao aumento do risco cardiovascular e a HAS acelera a morbidade e a mortalidade, marcadamente nesses pacientes (O'KEEFE JR et al., 1999). O estudo HDS (HYPERTENSION..., 1993) evidenciou que a associação, no mesmo paciente, de DM2 e HAS aumenta em aproximadamente quatro vezes o risco de complicações cardiovasculares, em relação à população normotensa não-diabética. Essa situação tornase ainda mais provável se estiver presente HVE, que é o mais temeroso fator de risco para insuficiência cardíaca, acidente vascular cerebral e coronariopatia (OIGMAN; NEVES, 1998).

Por essas razões, mas também devido à escassez de estudos semelhantes na região de Campina Grande (Paraíba), foi investigada a possível associação de HAS e HVE em pacientes com DM2 nessa região.

\section{MATERIAIS E MÉTODOS}

A população estudada foi constituída de pessoas com DM2, de ambos os sexos, atendidas no Ambulatório de Endocrinologia do Hospital Universitário Alcides Carneiro (HUAC) de Campina Grande (Paraíba), no período de abril de 2001 a março de 2002.

Os pacientes foram informados sobre esse estudo transversal e demonstraram entendimento quanto aos objetivos pretendidos; aqueles que concordaram em participar assinaram o termo de consentimento livre e esclarecido.

Somente foram incluídos no estudo os pacientes com diagnóstico prévio de DM2, portadores ou não de HAS, e excluídos os pacientes que não concordaram em assinar o termo de consentimento, assim como aqueles que apresentassem morbidades prévias associadas e que pudessem interferir na avaliação da HVE - antecedente de infarto do miocárdio, presença de bloqueios de ramos ao eletrocardiograma (ECG), alteraçōes eletrocardiográficas compatíveis com insuficiência coronariana, índice de massa corpórea $(\mathrm{IMC}) \geq 35 \mathrm{Kg} / \mathrm{m}^{2}$ e estenose aórtica ao ecocardiograma (ECO).

Após revisão do prontuário, avaliação clínico-cardiológica e aplicação dos critérios de exclusão, foram sorteados 48 pacientes, que foram convocados através do Serviço Social do HUAC a retornar ao ambulatório, com a finalidade de realizar o ECO. $\mathrm{Na}$ avaliação clínicocardiológica, foram estudadas ou descritas as seguintes variáveis: idade, gênero, procedência, grupo racial, tempo de evolução do DM2, uso de medicação anti-diabética, antecedente pessoal de HAS, tempo de evolução da HAS, uso de medicação anti-hipertensiva, tabagismo, IMC, medida da circunferência abdominal, medida da pressão arterial (PA), ECG $(\mathrm{n}=204)$ e ECO $(n=48)$.

O IMC foi calculado pela fórmula de Gray (1989), utilizando-se a classificação de obesidade adotada pelo III Consenso LatinoAmericano de Obesidade (1998) e a medida da circunferência abdominal foi expressa em centímetros. A verificação da PA foi realizada com esfigmomanômetro aneróide tipo padrão, previamente calibrado, na posição sentada e seguindo-se as recomendaçôes do sexto relatório do Joint National Committee (THE SIXTH..., 1997). Foi definido como hipertenso o indivíduo que apresentasse pressão arterial sistólica (PAS) $\geq 140 \mathrm{mmHg}$ e (ou) pressão arterial diastólica (PAD) $\geq 90 \mathrm{mmHg}$ (III CONSENSO..., 1998), ou o indivíduo sabidamente hipertenso, que vinha em uso de medicação antihipertensiva e que podia estar com níveis elevados ou não no momento da verificação da PA. Os eletrocardiogramas de 12 derivaçôes foram realizados por técnico especializado em eletrocardiografia e analisados pela autora. Para o diagnóstico de HVE ao ECG, foram observados os seguintes critérios de voltagem: índice 
de Sokolov-Lyon >35mm (SOKOLOV; LYON, 1949) e (ou) índice de Cornell $\geq 20 \mathrm{~mm}$ em mulheres e $28 \mathrm{~mm}$ em homens (CASALE et al., 1987). O ECO modo-M, bidimensional, "doppler" colorido, foi realizado em equipamento Vingemed ${ }^{\circledR}$, modelo "System Five", utilizando transdutor de $2,5 \mathrm{MHz}$, em posição de decúbito lateral esquerdo, com transdutor posicionado entre o terceiro e o quarto espaços intercostais esquerdos. As medidas cardíacas foram realizadas segundo recomendações da Sociedade Americana de Ecocardiografia (SANH et al., 1978). Para diagnóstico de HVE ao ECO, foram determinados a massa ventricular esquerda (MVE), o índice de massa ventricular esquerda (IMVE) e a espessura relativa de parede posterior (ERPP). Utilizando-se as fórmulas e parâmetros recomendados por Devereux e Reichek (1977), Savage e colaboradores (1987) e Devereux e colaboradores (1984) considerouse como tendo HVE os pacientes que apresentassem IMVE $>131 \mathrm{~g} / \mathrm{m}^{2}$ no sexo masculino e $100 \mathrm{~g} / \mathrm{m}^{2}$ no sexo feminino e/ou ERPP $>0,44$.

Foi realizada a análise descritiva dos resultados, tendo como variáveis-respostas a freqüência de HAS e HVE na amostra estudada. As variáveis contínuas descritas como média \pm desvio padrão e as variáveis categóricas, expressas como proporções, foram comparadas, respectivamente, pelo teste t de Student, o teste do qui-quadrado e o teste exato de Fisher, com intervalo de confiança de $95 \%$ e nível de significância $(p)<0,05$.

\section{RESULTADOS}

Foram examinados 262 pacientes e, depois de aplicados os critérios de exclusão, foram alocadas 204 pessoas no estudo. As características demográficas da população estudada estão apresentadas na Tabela 1 .

No exame, utilizando-se os critérios estabelecidos para diagnóstico de HAS, observouse sua presença em $136(66,7 \%)$ pacientes, enquanto $68(33,3 \%)$ eram não-hipertensos.

Entre os $136(66,7 \% / 204)$ pacientes hipertensos encontrados, cento e três $(50,5 \% /$
204) já relatavam antecedente pessoal de HAS, com tempo médio de evolução de $88,7 \pm 74,3$ meses, e $81,5 \%(84 / 103)$ utilizavam terapia anti-hipertensiva, enquanto $18,5 \%(19 / 103)$ não estavam em uso de medicação alguma, ou o uso era irregular.

Tabela 1 - Características demográficas

\begin{tabular}{ccc}
\hline Características & Número & $\%$ \\
\hline IDADE (anos) $58 \pm 11,4$ & & \\
GÊNERO: & 131 & 64,2 \\
Feminino & 73 & 35,8 \\
Masculino & & \\
PROCEDÊNCIA: & 125 & 61,3 \\
Campina Grande & 79 & 38,7 \\
Outras localidades & & \\
GRUPO RACIAL: & 125 & 61,3 \\
Mulato & 58 & 28,4 \\
Branco & 18 & 8,8 \\
Negro & 3 & 1,5 \\
Mestiço de índio & &
\end{tabular}

Avaliando-se as características clínicas da população estudada, observou-se que os pacientes diabéticos hipertensos tinham significativamente mais idade $(p<0,0001)$, maior tempo de evolução do diabetes $(\mathrm{p}<0,015)$ e maior circunferência abdominal $(\mathrm{p}<0,008)$ que os nãohipertensos, como se mostra na Tabela 2.

Estudando-se a associação de HAS com outras variáveis (gênero, grupo racial, tabagismo, IMC e uso de medicação anti-diabética), observou-se que a hipertensão foi mais freqüente no gênero feminino $(69,5 \%)$, em indivíduos do grupo racial branco $(72,4 \%)$, ex-tabagistas $(71,3 \%)$, com sobrepeso $(69,3 \%)$ e em uso de insulina associada a hipoglicemiante oral (80\%). Entretanto, entre essas variáveis, apenas o tabagismo apresentou relação estatisticamente significante $(p<0,009)$ com a presença de HAS (TABELA 3). 
Tabela 2 - Características clínicas e sua relação com HAS

\begin{tabular}{lcccc}
\hline \multicolumn{5}{c}{ HAS } \\
\hline Características & Total (204) & $\begin{array}{c}\text { Presença } \\
(136)\end{array}$ & $\begin{array}{c}\text { Ausência } \\
(68)\end{array}$ & p valor \\
Idade (anos) & $58,0 \pm 11,4$ & $61,5 \pm 10,1$ & $51,0 \pm 10,6$ & $<0,0001(1)$ \\
$\begin{array}{l}\text { Tempo de diabetes } \\
\text { (meses) }\end{array}$ & $86,9 \pm 78,4$ & $96,4 \pm 84,4$ & $67,8 \pm 60,9$ & $<0,015(1)$ \\
$\begin{array}{l}\text { PAS sentada } \\
\text { PAD sentada }\end{array}$ & $85,2 \pm 13,5$ & $89,9 \pm 13,4$ & $75,7 \pm 7,4$ & $\mathrm{~ns}$ \\
$\begin{array}{l}\text { Circunferência } \\
\text { abdominal (cm) }\end{array}$ & $92,5 \pm 9,6$ & $93,8 \pm 9,3$ & $90,0 \pm 10,0$ & $<0,008(1)$ \\
\hline $\begin{array}{l}\text { Nota: ns= não significante; (1) Teste t de Student } \\
\text { (14) }\end{array}$ & & & & \\
\hline
\end{tabular}

Tabela 3 - Associação da HAS a outros fatores de risco

\begin{tabular}{|c|c|c|c|c|}
\hline \multirow[b]{2}{*}{ Variáveis } & \multicolumn{4}{|c|}{ HAS } \\
\hline & $\begin{array}{l}\text { n Total } \\
(\mathrm{n}=204)\end{array}$ & $\begin{array}{l}\text { Presente } \\
(\mathrm{n}=136)\end{array}$ & $\begin{array}{c}\text { Ausente } \\
(\mathrm{n}=68)\end{array}$ & $p$ valor \\
\hline \multicolumn{5}{|l|}{ GÊNERO, $\mathrm{n}(\%)$} \\
\hline Feminino & $131(64,2)$ & $91(69,5)$ & $40(30,5)$ & $>0,25(1)$ \\
\hline Masculino & $73(35,8)$ & $45(61,6)$ & $28(38,4)$ & \\
\hline \multicolumn{5}{|l|}{ GRUPO RACIAL, n(\%) } \\
\hline Branco & $58(28,4)$ & $42(72,4)$ & $16(27,6)$ & $>0,26(1)$ \\
\hline Não-branco & $146(71,6)$ & $94(64,4)$ & $52(35,6)$ & \\
\hline \multicolumn{5}{|l|}{ TABAGISMO, $\mathrm{n}(\%)$} \\
\hline Não & $79(38,7)$ & $54(68,4)$ & $25(31,6)$ & \\
\hline Sim & $32(15,7)$ & $14(43,8)$ & $18(56,2)$ & $<0,009$ (1) \\
\hline Ex-Tabagista & $93(45,6)$ & $68(73.1)$ & $25(29,9)$ & \\
\hline \multicolumn{5}{|l|}{ TRATAMENTO DIABETES, n(\%) } \\
\hline Não & $37(18,1)$ & $25(67,5)$ & $12(32,5)$ & \\
\hline Anti-diabetico oral & $80(39,2)$ & $55(68,7)$ & $25(31,3)$ & $>0,75(1)$ \\
\hline Insulina & $77(37,8)$ & $48(55,1)$ & $29(44,9)$ & \\
\hline Insulina + anti-diabético oral & $10(4,9)$ & $8(80,8)$ & $2(20,0)$ & \\
\hline \multicolumn{5}{|l|}{$\operatorname{IMC}\left(\mathrm{Kg} / \mathrm{m}^{2}\right), \mathrm{n}(\%)$} \\
\hline Normal + Baixo peso $(n=2)(1)$ & $66(32,4)$ & 41 & $25(37,9)$ & \\
\hline Sobrepeso & $101(49,5)$ & $(62,1)(2)$ & $31(30,7)$ & $>0,61(1)$ \\
\hline \multirow[t]{2}{*}{ Obeso (I) } & $37(18,1)$ & $70(69,3)$ & $12(32,4)$ & \\
\hline & & $25(67,6)$ & & \\
\hline
\end{tabular}

(1) Teste do qui-quadrado. (2) 2 pessoas com baixo peso 
A HVE estava presente ao ECG em $11,3 \% \quad(n=23 / 204)$ dos pacientes. O ecocardiograma foi realizado em 48 (23,5\%) indivíduos, porém um foi excluído, por apresentar estenose aórtica não detectada anteriormente. A média de IMVE nos 47 pacientes estudados foi de $106,3 \pm 32,1 \mathrm{~g} / \mathrm{m}^{2}$, com o mínimo de $54,7 \mathrm{~g} / \mathrm{m}^{2}$ e o máximo de $198,3 \mathrm{~g} / \mathrm{m}^{2}$. A
ERPP média encontrada foi $0,50 \pm 0,10$, com o mínimo de 0,35 e máximo de 0,66. Observouse a presença de HVE em 76,6\% (36/47) dos pacientes avaliados e em 85,2 (23/27) dos pacientes hipertensos; no entanto não existiu relação estatisticamente significante entre as duas variáveis (TABELA 4).

Tabela 4 - Relação entre HAS e HVE ao Ecocardiograma ( $n=47)$

\begin{tabular}{lcccr}
\hline \multicolumn{5}{c}{ HVE, n $(\%)$} \\
\hline HAS & SIM & NĀO & TOTAL & $p$ valor \\
SIM & $23(85,2)$ & $4(14,8)$ & $27(57,5)$ & $>0,19(1)$ \\
NÃO & $13(65,0)$ & $7(35,0)$ & $20(42,5)$ & \\
TOTAL & $36(76,6)$ & $11(23,4)$ & $47(100,0)$ & \\
\hline
\end{tabular}

(1)Teste exato de Fisher

Entre os 47 casos com ECG e ECO, a distribuição do diagnóstico de HVE foi: a) 2 $(4,3 \%)$ casos com HVE em ambos os exames; b) nenhum caso de HVE apenas pelo ECG; c) $34(72,3 \%)$ casos com HVE somente pelo ECO e d) $11(23,4 \%)$ casos sem HVE por ambos os exames. Desse modo, esses resultados tiveram o índice Kappa de 0,02, o que corresponde a uma concordância fraca.

\section{DISCUSSÃO}

A prevalência de $66,7 \%$ para HAS entre pacientes com DM2 é superior à encontrada pelo HDS (HYPERTENSION..., 1993), que foi de $40 \%$. Essa diferença deve-se provavelmente ao fato de que, nesse estudo, utilizaram-se como parâmetros, níveis superiores de $\mathrm{PA}(\geq 160 \mathrm{x}$ $90 \mathrm{mmHg}$ ), bem como às características da população estudada, uma vez que Fonseca e colaboradores. (1995) e Oliveira e colaboradores. (1998) encontraram prevalências de $60,1 \%$ e $64,7 \%$ respectivamente, em pacientes brasileiros.

A HAS foi mais freqüente nas mulheres. A idade mostrou-se um importante fator associado $(p<0,0001)$ à HAS, possivelmente em decorrência do processo aterosclerótico que, in- clusive, é mais prevalente em pacientes diabéticos (BLOOMGARDEN, 2000), com maior acometimento do gênero feminino (SMITHERMAN; REIS, 1997). O tempo de evolução DM2 foi outro importante fator relacionado à HAS $(p<0,015)$, resultado discordante daquele encontrado por Fonseca e colaboradores (1995), que observaram relação com a idade do paciente, mas não com o tempo do surgimento do DM2.

Vários estudos têm descrito a obesidade e, em particular, a obesidade central, como um importante determinante de HAS (POULIOT et al., 1994). No presente trabalho, observouse que os pacientes que apresentavam sobrepeso e obesidade grau I, assim como maior aumento da circunferência abdominal, tiveram maior prevalência de HAS, porém sendo estatisticamente significante apenas em relação a essa última $(p<0,008)$. Não obstante, isso provavelmente decorreu do processo de seleção, uma vez que foram excluídos pacientes com IMC $\geq 35$ $\mathrm{Kg} / \mathrm{m}^{2}$, tendo em vista que níveis mais elevados de IMC podem levar à HVE, mesmo em pacientes normotensos e não-diabéticos (CRISOSTOMO et al., 2001). Chama a atenção a ocorrência de hipertensão nos dois pacientes com baixo peso, o que sugere ser a resistência à insulina a principal responsável pela 
HAS em diabéticos (OSEI, 1999).

$\mathrm{O}$ uso do tabaco está relacionado à elevação aguda da PA (BENOWITZ; KUYT; JACOB, 1984). Paradoxalmente, neste trabalho, ao se associar HAS ao tabagismo, observouse que os pacientes tabagistas apresentavam menor freqüência de HAS. Resultados semelhantes foram encontrados por Goldbourt e Medalie (1997). Nos ex-tabagistas, a freqüência de HAS foi discretamente superior à dos não-tabagistas, fato corroborado por Green, Jucha e Luz (1986), os quais observaram que a eliminação do hábito de fumar pode, inclusive, levar ao aumento da $\mathrm{PA}$, e que provavelmente tem relação com o ganho de peso nesses indivíduos. Em todo caso, esses resultados devem ser analisados com cautela e talvez indiquem os efeitos de variáveis não estudadas e (ou) mesmo a complexa e multifatorial etiologia da HAS. Por isso, os estudos sobre os diversos efeitos do tabagismo no sistema cardiovascular devem merecer análises multivariadas e estudos de meta-análise.

A HVE é um dos mais importantes fatores de risco independentes de doença cardiovascular. A HAS arterial permanece como o fator de risco mais comum de complicações cardiovasculares e a presença de HVE aumenta tal risco (OIGMAN; NEVES, 2000). Este estudo demonstra que a HVE é mais prevalente em diabéticos hipertensos que nos nãohipertensos, à semelhança dos resultados obtidos no estudo HyperGEN (PALMIERI et al., 2001), estando presente em $85,2 \%$ dos diabéticos hipertensos, sendo superior aos resultados (72\%) encontrados por Grossman e colaboradores (1992), provavelmente porque, nesse último, foi considerado como parâmetro apenas o IMVE, sem levar em consideração a ERPP. Além do mais, os valores considerados (IMVE > 134 $\mathrm{g} / \mathrm{m}^{2}$ em homens e $110 \mathrm{~g} / \mathrm{m}^{2}$ em mulheres) foram superiores aos aqui empregados. Apesar disso, não houve associação significante do ponto de vista estatístico $(p>0,19)$ entre HAS e HVE, possivelmente até pelo efeito do tamanho amostral $(n=47)$. Também se deve considerar o fato de 84 pacientes hipertensos estarem em uso de medicação anti-hipertensiva, o que pode ter contribuído para mascarar aquela associação, uma vez que estudos têm demonstrado que a terapia anti-hipertensiva pode levar à regressão da HVE (DAHLÖF; PENNERT; HANSSON, 1992; JENNINGS; WONG, 1998). De outro lado, chama a atenção o fato de que $65 \%$ dos diabéticos não hipertensos apresentam também a HVE, o que pode estar relacionado à existência de uma cardiomiopatia inerente ao diabetes (GALDERISI et al., 1991).

Embora o ECG tenha mostrado uma prevalência $(11,3 \%)$ de HVE superior àquela descrita por Leonetti e Cuspidi (1995) em hipertensos não diabéticos ( $3 \%$ a $5 \%$ ), o ECO mostrou ser um melhor exame para diagnóstico de HVE, em consonância com os estudos de Levy (1988) e Leonetti e Cuspidi (1995).

\section{CONCLUSÃO}

Em conclusão, entre os 204 pacientes com DM2 estudados, a elevada prevalência de HAS, associada à idade, ao tempo de evolução do diabetes, ao aumento da circunferência abdominal e ao tabagismo, evidencia a relevância clínica da avaliação diferenciada desses pacientes. Embora os indivíduos hipertensos tivessem maior prevalência de HVE, esta também foi elevada entre os diabéticos não hipertensos, o que sugere a existência de cardiomiopatia associada ao DM2. Por isso, a realização do ECO, deve ser sempre avaliada, para diagnóstico e tratamento precoce desse importante fator de risco.

\title{
Arterial hypertension and left ventricular hypertrophy in type 2 diabetes mellitus
}

\author{
Abstract \\ In an attempt to evaluate the prevalence of Systemic Arterial Hypertension (SAH), Left Ventricular Hypertrophy \\ $(L V H)$ as well as their association with Type 2 Diabetes Mellitus, 204 patients were studied at Hospital
}


Universitário Alcides Carneiro (HUAC) at Universidade Federal de Campina Grande - PB. The group's average age was $58.0 \pm 11.4$ years, and $64.2 \%(n=131 / 204)$ were female. SAH was observed in $66.7 \%$ $(n=136 / 204)$ of the group, and it been found more often in women (69.5\%), in white people and former smokers. A statistically significant association was found between $S A H$ and age $(p<0.0001)$, diabetes evolution time $(p<0.015)$, smoking $(p<0.009)$ and enlargement of abdominal circumference $(p<0.008)$. LVH was found in $11.3 \%$ (23/204) of the patients analyzed by electrocardiogram (ECG). The echocardiogram performed in 23.0\% (47/204) of patients of the same group showed a LVH prevalence of $76.6 \%$ (36/47). Although it was found a higher incidence of LVH in hypertensives (85.2\%) than in patients with normal blood pressure (65.0\%), no statistical correlation was found among those variables in the group studied. As a conclusion, the prevalence of SAH was $66.7 \%$ in the group studied. A correlation between SAH and age, evolution time of diabetes, enlargement of abdominal circumference, and smoking was found. There was also a high prevalence of LVH (76.6\%) when analyzed through echocardiogram, although, no correlation was found between SAH and LVH.

Keywords: arterial hypertension; left ventricular hypertrophy; diabetes, type 2.

\section{REFERÊNCIAS}

BENOWITZ, N.L.; KUYT, F.; JACOB, P. Influence of nicotine on cardiovascular and hormonal effects of smoking. Clin. Pharmacol. Ther., St. Louis, v.36, p.74, 1984.

BLOOMGARDEN, Z.T. American Diabetes Association Annual Meeting, 1999.

Diabetes Care, Alexandria, v.23, p.845-852, 2000.

BRANDÃO, A.P. et al. Hipertensão arterial. Disponível em: <http://www.lampada.uerj.br/ eadind/has/has.html>. Acesso em: 26 nov. 2000.

CASALE, P.N. et al. Improved sex-specific criteria of left ventricular hypertrophy for clinical and computer interpretation of electrocardiograms: validation with autopsy findings. Circulation, Hagerstown, v.75, p.565572, 1987.

III CONSENSO Brasileiro de Hipertensão Arterial. Rev. Bras. Clin. Terap., São Paulo, v.24, n.6, p.231-272, 1998.

III CONSENSO Latino-Americano de Obesidade. 1998. Disponível em: < $\underline{\text { http:// }}$ ww.abeso.org.br>. Acesso em: 22 fev. 2002.
CONTI, C.R. Diabetes, hypertension and cardiovascular disease. Clin. Cardiol., Mahwah, v.21, p.1, 2001.

CRISOSTOMO, L.L. et al. Left ventricular mass and function in young obese women. Int. J. Obes. Relat. Metab. Disord., v.25, n.2, p.233-238, Feb. 2001.

DAHLÖF, B.; PENNERT, K.; HANSSON, L. Regression of left ventricular hypertrophy: a meta-analysis. Am. J. Hypertens., New York, v.5, p.95-110, 1992.

DEVEREUX, R.B. et al. Standardization of M-mode echocardiographic left ventricular anatomic measurements. J. Am. Coll. Cardiol., New York, v.4, p.1222-1230, 1984.

DEVEREUX, R.B.; REICHEK, N. Echocardiographic determination of left ventricular mass in man: anatomic validation of the method. Circulation, Hagerstown, v.55, p.613-618, 1977.

FONSECA, C.T. et al. Prevalência de hipertensão arterial entre diabéticos do tipo I e tipo II em nível terciário de atenção. R. Asoc. Latinoam. Diab., Buenos Aires, v.3, p.85, 1995. 
GALDERISI, M. et al. Echocardiographic evidence for existence of a distinct diabetic cardiomyopathy (The Framingham Heart Study). Am. J. Cardiol., New York, v.68, p.8589, 1991.

GOLDBOURT, U.; MEDALIE, J.H. Characteristics of smokers, non-smokers and exsmokers among 10,000 adult males in Israel. II. Physiologic, biochemical and genetic characteristics. Am. J. Epidemiol., Cary, v.105, p.75-86, 1977.

GRAY, D.S. Diagnosis and prevalence of obesity. Med. Clin. North Am., Philadelphia, v.73, p.1-14, 1989.

GREEN, M.S.; JUCHA, E.; LUZ, Y. Blood pressure in smokers and non-smokers: epidemiologic findings. Am. Heart J., St. Louis, v.111, p.932-940, 1986.

GROSSMAN, E. et al. Left ventricular mass in diabetes-hypertension. Arch. Intern. Med., Chicago, v.152, p.1001-1004, 1992.

HYPERTENSION in Diabetes Study (HDS): I. Prevalence of hypertension in newly presenting type 2 diabetic patients and the association with risk factors for cardiovascular and diabetic complications. J. Hypertens., London, v.11, p.309-317, 1993.

JENNINGS, G.; WONG, J. Regression of left ventricular hypertrophy in hypertension: changing patterns with sucessive meta-analysis. J. Hypertens., London, v.16, p.S29-34, 1998.

LEONETTI, G.; CUSPIDI, C. The heart and vascular changes in hypertension. J. Hypertens., London, v.13, p.S29-33, 1995.

LEVY, D. Left ventricular hypertrophy: epidemiological insights from the Framingham Heart Study. Drugs, Auckland, v.35, p.1-5, 1988. Suppl. 1.

MALERBI, D.A ; FRANCO, L.J. Multicenter study of the prevalence of diabetes mellitus and impaired glucose tolerance in the urban Brazilian population aged 30-69yr: The Brazilian Cooperative Group on the Study of Diabetes Prevalence. Diabetes Care, Alexandria, v.15, p.1509-1516, 1992.
OIGMAN, W.; NEVES, M.F.P. Hipertensão arterial e hipertrofia ventricular esquerda: epidemiologia e diagnóstico. Hipertensão, São Paulo, v.1, p.140-145, 1998.

OIGMAN, W.; NEVES, M.F.P. Sistema reninaangiotensina e hipertrofia ventricular esquerda. R. Bras. Hiperten., São Paulo, v.7, p.261-267, 2000 .

O'KEEFE JR, J.H. et al. Improving the adverse cardiovascular prognosis of type 2 diabetes. Mayo Clin. Proc., Rochester, v.74, p.171-180, 1999.

OLIVEIRA, J.E.P. et al. Tratamento do diabetes mellitus entre 1991-1995: nível de controle e complicaçôes. R. Asoc. Latinoam. Diab., Buenos Aires, v.6, p.122, 1998.

OSEI, K. Insulin resistence and systemic hypertension. Am. J. Cardiol., New York, v.84, p.33-36, 1999.

PALMIERI, V. et al. Effect of type 2 diabetes mellitus on left ventricular geometry and systolic function in hypertensive subjects: Hypertension Genetic Epidemiology Network (HyperGEN) Study. Circulation, Hagerstown, v.103, p.101107, 2001.

POULIOT, M.C. et al. Waist circumference and abdominal saggital diameter: best simple anthropometric indexes of abdominal visceral adipose accumulation and related cardiovascular risk in men and women. Am. J. Cardiol., New York, v.73, p.460-468, 1994.

SANH, D.J. et al. Recomendations regarding quantification in M-mode echocardiography: results of a survey of echocardiographic measurements. Circulation, Hagerstown, v.58, p.1072-1083, 1978.

SAVAGE, D.D. et al. The spectrum of left ventricular hypertrophy in a general population sample: The Framingham Study. Circulation, Hagerstown, v.75, n.1, pt.2, p.I26-33, 1987.

THE SIXTH report of the Joint National Committee on prevention, detection, evaluation, and treatment of high blood pressure. Arch. Intern. Med., Chicago, v.157, p.24132446, 1997. 
SMITHERMAN, T.C.; REIS, S.E. Heart disease in women with diabetes. Diabetes Spectrum, Alexandria, v.10, p.265-276, 1997. SOKOLOV, M.; LYON, T.P. The ventricular complex in left ventricular hypertrophy as obtained by unipolar precordial and limb leads. Am. Heart J., St. Louis, v.37, p.161-186, 1949.
WEIDMANN, P.; BOEHLEN, L.M.; DE COURTEN, M. The pathogenesis and treatment of hypertension associated with diabetes mellitus. Am. Heart J., St. Louis, v.125, p.1498-1513, 1993.

Recebido em / Received: 19/07/2004 Aceito em / Accepted: 23/11/2004 